\title{
Dramatic Neuronal Rescue with Prolonged Selective Head Cooling after Ischemia in Fetal Lambs
}

\author{
Alistair J. Gunn, Tania R. Gunn, Harmen H. de Haan, Christopher E. Williams, and Peter D. Gluckman \\ Research Centre for Developmental Medicine and Biology, Department of Paediatrics, School of Medicine, University of Auckland, \\ Auckland, New Zealand
}

\begin{abstract}
Hypothermia has been proposed as a neuroprotective strategy. However, short-term cooling after hypoxia-ischemia is effective only if started immediately during resuscitation. The aim of this study was to determine whether prolonged head cooling, delayed into the late postinsult period, improves outcome from severe ischemia. Unanesthetized near term fetal sheep were subject to $30 \mathrm{~min}$ of cerebral ischemia. 90 min later they were randomized to either cooling $(n=9)$ or sham cooling $(n=7)$ for $72 \mathrm{~h}$. Intrauterine cooling was induced by a coil around the fetal head, leading initially to a fall in extradural temperature of $5-10^{\circ} \mathrm{C}$, and a fall in esophageal temperature of $1.5-3^{\circ} \mathrm{C}$. Cooling was associated with mild transient systemic metabolic effects, but not with hypotension or altered fetal heart rate. Cerebral cooling reduced secondary cortical cytotoxic edema $(P<0.001)$. After $5 \mathrm{~d}$ of recovery there was greater residual electroencephalogram activity $(-5.2 \pm 1.6$ vs. $-15.5 \pm 1.5 \mathrm{~dB}, P<0.001)$ and a dramatic reduction in the extent of cortical infarction and neuronal loss in all regions assessed (e.g., 40 vs. $99 \%$ in the parasagittal cortex, $P<0.001)$. Selective head cooling, maintained throughout the secondary phase of injury, is noninvasive and safe and shows potential for improving neonatal outcome after perinatal asphyxia. (J. Clin. Invest. 1997. 99:248-256.) Key words: hypothermia-induced - cerebral ischemia therapy - hypoxic-ischemic encephalopathy • fetal monitoring $\bullet$ fetal lamb
\end{abstract}

\section{Introduction}

Even small changes in temperature during experimental hypoxia-ischemia critically modulate neural outcome (1). This effect, which appears to be disproportionate to the known changes in cerebral metabolism, is postulated to be related in part to a reduction in damaging events such as the release of excitotoxins and oxygen free radicals $(2,3)$. In contrast however, studies of brief hypothermia during recovery from hypoxia-ischemia in neonatal animals have produced inconsis-

Address correspondence to Dr. Alistair Gunn, Research Centre for Developmental Medicine and Biology, Department of Paediatrics, School of Medicine, University of Auckland, Private Bag 92019, Auckland, New Zealand. Phone: 64-9-3737599 x6763; FAX: 64-93737497; E-mail: aj.gunn@auckland.ac.nz

Received for publication 2 April 1996 and accepted in revised form 11 November 1996.

J. Clin. Invest.

(C) The American Society for Clinical Investigation, Inc.

0021-9738/97/01/248/09 \$2.00

Volume 99, Number 2, January 1997, 248-256 tent results $(4,5)$. Similar studies in adult animals suggest that cooling is most effective after relatively mild insults $(6,7)$, and that the therapeutic window after reperfusion during which cooling remains effective is very narrow (8-11). Although more prolonged cooling, for 5-24 h $(7,10,12,13)$, appears to be more consistently effective, studies to date have not logically related the period of cooling to the pathophysiological phases of neural injury.

Clinical and experimental studies suggest that as well as a primary phase of energy failure during hypoxia-ischemia, secondary energy failure occurs $8-48 \mathrm{~h}$ later $(13,14)$, which in the human infant is associated with poor neurodevelopment at 1 $\mathrm{yr}$ of age (14). We have demonstrated that, after $30 \mathrm{~min}$ of ischemia in the near term fetal sheep, delayed seizures and cytotoxic cell swelling occur in this secondary phase (15). This is paralleled by accumulation of excitotoxins (16) and by vasodilation (17).

We hypothesized that delayed postinsult hypothermia maintained throughout the course of the secondary phase would alleviate membrane dysfunction and reduce secondary neuronal damage. By selectively cooling the head and keeping systemic hypothermia to a minimum, the unwanted systemic complications of hypothermia $(18,19)$ should be minimized.

\section{Methods}

Surgical procedures. Studies were approved by the Animal Ethics Committee of the University of Auckland. Romney/Suffolk fetal sheep from 117 to $124 \mathrm{~d}$ of gestation were operated on under $2 \%$ halothane/oxygen anesthesia as described previously $(15,20)$. Polyvinyl catheters were inserted into both fetal brachial arteries and the amniotic sac. The vertebral-occipital anastomoses were ligated bilaterally to restrict vertebral blood supply to the carotid arteries; the lingual arteries were also ligated to restrict non-cerebral blood flow (21). A double ballooned inflatable occluder cuff was placed around each carotid artery. A 3-mm ultrasonic flow probe (Transonic Systems Inc., Ithaca, NY) was placed on each carotid artery just proximal to the cuff. Two pairs of electroencephalographic (EEG) ${ }^{1}$ electrodes (AS633-5SSF; Cooner Wire Co., Chatsworth, CA) were placed on the dura over the parasagittal parietal cortex $(5 \mathrm{~mm}$ and $15 \mathrm{~mm}$ anterior to bregma and $10 \mathrm{~mm}$ lateral), with a reference electrode sewn over the occiput. To record cortical impedance, a third pair of electrodes (AS633-3SSF; Cooner Wire Co.) was placed over the dura, $5 \mathrm{~mm}$ lateral to the EEG electrodes. A thermistor (Incu-Temp-1; Mallinckrodt Medical Inc., St. Louis, MO) was placed over the parasagittal dura $20 \mathrm{~mm}$ anterior to bregma. A 20-gauge catheter was placed in the sagittal sinus through a shallow midline burr hole. The burr holes were sealed, and the skin over the fetal skull was closed, using cyanoacrylate glue. A "cooling" coil made from silastic tubing (external diameter: $7.9 \mathrm{~mm}$; internal diameter: $4.8 \mathrm{~mm}$; Silclear; Degania Sili-

1. Abbreviations used in this paper: $\mathrm{CBF}$, cerebral blood flow; $\mathrm{CMRO}_{2}$, cerebral metabolic rate for oxygen; EEG, electroencephalogram. 
cone, Degania Bet, Israel) was attached over the scalp and wrapped around down to the level of the external auditory meatus. A second thermistor (to measure fetal core body temperature) was placed in the fetal esophagus, at the level of the right atrium (19). The fetus was then returned to the uterus, and all leads and catheters were exteriorized to the maternal flank. A polyvinyl catheter and a third thermistor were advanced $20 \mathrm{~cm}$ into the maternal tarsal vein.

After the operation, the ewes were housed in metabolic cages at constant temperature $\left(16 \pm 1^{\circ} \mathrm{C}\right)$ and humidity $(50 \pm 10 \%)$, in a $12-\mathrm{h}$ light/dark cycle, with free access to hay and water, supplemented by sheep nuts and alfalfa. Gentamicin, $80 \mathrm{mg}$, was administered daily to the ewe, intravenously, for $5 \mathrm{~d}$ starting at surgery.

Recordings. Fetal arterial blood pressure, corrected for amniotic fluid pressure (Novatrans II, MX860; Medex Inc., Hilliard, OH), carotid arterial blood flows (T208 Ultrasonic Flowmeter; Transonic Systems Inc.), fetal extradural temperature, esophageal temperature, and maternal temperature, as well as the fetal parietal EEG and impedance, were recorded continuously. Recordings started $24 \mathrm{~h}$ before the experiment and continued for $120 \mathrm{~h}$ afterwards. Signals were averaged at 1-min intervals and stored on disk by custom software (Labview for Windows; National Instruments Ltd., Austin, TX), running on an IBM-compatible computer. The EEG signal was low pass filtered at $30 \mathrm{~Hz}$, and then the intensity spectrum and impedance signal were extracted as reported previously $(15,17,22)$. Cortical impedance was measured using a four electrode technique (15); the impedance of a tissue rises concomitantly as cells depolarize and fluid shifts from the extracellular to the intracellular space and cytotoxic edema develops $(15,16)$. The raw EEG signal was recorded for off-line detection of seizure events using commercial software that analyses spike wave and polyspike activity (Monitor; Stellate Systems, Quebec, Canada). For automatic detection, seizures were defined according to the default settings of Monitor software (17).

Experimental procedures. Before experimentation, arterial blood samples were analyzed for blood gases, $\mathrm{pH}$ (Radiometer ABL 330, Copenhagen, Denmark), oxygen saturation, hemoglobin, glucose, and lactate. After $24 \mathrm{~h}$ of baseline recordings, reversible cerebral ischemia was induced by inflating the carotid cuffs bilaterally with sterile saline for $30 \mathrm{~min}$. Successful occlusion was confirmed by the onset of an isoelectric EEG signal within $30 \mathrm{~s}$ of inflation. Fetal arterial and sagittal sinus blood samples were drawn $60 \mathrm{~min}$ and just before occlusion and then at $35 \mathrm{~min}$ after occlusion (i.e., $5 \mathrm{~min}$ after the end of occlusion), $2 \mathrm{~h}$ (just before cooling), $2.5 \mathrm{~h}, 4 \mathrm{~h}, 8 \mathrm{~h}, 24 \mathrm{~h}, 48 \mathrm{~h}, 72 \mathrm{~h}, 96 \mathrm{~h}$, and $120 \mathrm{~h}$, and analyzed for $\mathrm{pH}, \mathrm{PaO}_{2}, \mathrm{pCO}_{2}$, lactate, and glucose levels.

Fetuses were randomized to either cooling or sham cooling, starting 90 min after reperfusion and continuing until $72 \mathrm{~h}$ after occlusion. Cooling was performed by linking the cooling coil over the fetal scalp with a pump in a cooled water bath. In sham cooled fetuses, the water was not circulated; thus the cooling coil and its contents will have remained in equilibrium with fetal temperature. In the hypothermia group, the water bath temperature was $4^{\circ} \mathrm{C}$ at the start of cooling, and gradually warmed up to $\sim 14^{\circ} \mathrm{C}$ over the first day of cooling. Thereafter, water bath temperature remained stable until cooling was stopped. The fetuses were monitored for a further $48 \mathrm{~h}$, and then the ewe and fetus were killed by barbiturate overdose.

Histological analysis was performed as described previously (15, $20,22)$. The fetal brain was perfusion fixed in situ with $10 \%$ phosphate-buffered formalin. Neuronal loss was scored by light microscopy on $8-\mu \mathrm{m}$-thick coronal sections stained with thionin and acid fuchsin, by two assessors, one of whom was blinded to the treatment group. The proportion of neurons showing ischemic cell change in preassigned areas was scored on a 6 point scale: $0=$ no dead neurons; $5=>0-10 \% ; 30=>10-50 \% ; 70=>50-90 \% ; 95=90$ to $<100 \%$; $100=100 \%$ dead neurons. Average scores were calculated for each region. The proportion of areas within the parasagittal cortex showing infarction, with evidence of death of glia, as well as neurons (20), was also recorded.

Data analysis and statistics. Off-line analysis of the recorded signals was performed using a scientific software package (Viewdac
Data Acquisition; Keithley Instruments Inc., Taunton, MA). Time series analysis of the postreperfusion data, e.g., to calculate the magnitude and timing of peaks or troughs, was performed on the recorded signals smoothed with a digital median filter, to minimize the effects of short-term $(<20 \mathrm{~min})$ fluctuations. Average values for predetermined intervals were calculated: $-24-0 \mathrm{~h}, 0-30 \mathrm{~min}, 30-60 \mathrm{~min}, 1-2 \mathrm{~h}$, 2-4 h, 4-8 h, 8-12 h, 12-18 h, 18-24 h, then 12-h intervals up to $120 \mathrm{~h}$ after carotid occlusion. Data have been plotted using the midpoint of each interval, except for the 24-h baseline period data which have been plotted at $1 \mathrm{~h}$ before occlusion; additionally, to confirm that there had been no baseline drift, average values for the hour before occlusion have been presented at $0 \mathrm{~h}$.

The total EEG intensity (power) was log transformed [dB, $20 \times$ $\log ($ intensity)], as this transformation gives a better approximation to the normal distribution (23). The intensity was then normalized with respect to the 24-h baseline period: thus, all measurements are expressed as a ratio of the baseline period. The "final EEG intensity" was calculated on the final 12 -h period, 108-120 h. The suppression time of the EEG was defined as the time after insult during which the (smoothed) EEG intensity was $>5 \mathrm{~dB}$ below the baseline intensity. The spectral edge of the EEG was calculated from the spectra, as the frequency below which lay $90 \%$ of the EEG intensity.

The heights of the primary and secondary cortical impedance peaks were measured. The time for the primary peak to resolve to trough, defined as the $90 \%$ point of fall, and the time of the secondary peak were also determined. Impedance rises as the temperature of the medium, through which the signal passes, falls (24). Therefore, in each fetus, we measured the slope of impedance changes during a 30-min period of cooling before the start of baseline recording. The correlation between temperature and impedance was very good in each fetus: $r^{2} \geq 0.98$. The measured slope was used to correct the impedance signal for the effect of temperature changes during cooling: Corrected impedance $=$ impedance $-($ slope $\times \Delta$ temperature $)$. The corrected impedance data are presented as percentage of baseline.

Cerebral blood flow (CBF) was calculated as the sum of the left and right carotid blood flows. Since the vertebro-carotid anastomoses were ligated, all blood flow to the cortex and midbrain structures was derived from the carotid circulation (21). The period of secondary hypoperfusion was defined as the period from the time CBF fell below $90 \%$ of baseline, until the time it rose to $>90 \%$ of baseline. Cerebral metabolic rate for oxygen $\left(\mathrm{CMRO}_{2}\right)$ was determined from the difference in the oxygen contents of paired brachial artery and sagittal sinus blood samples, multiplied by the CBF at the time the samples were drawn: $\mathrm{CMRO}_{2}=($ arterial oxygen content - sagittal sinus oxygen content) $\times C B F$.

The effect of cooling on neuronal loss and other biophysical parameters was determined by multivariate analysis of variance (MANOVA). Cerebral region and time were treated as repeated measures, to allow for repeated sampling. For comparisons over time, baseline levels were used as a covariate (MANCOVA). Where significant differences were found, post-hoc comparisons of the means were made using the protected least significant difference test, adjusted using the baseline levels as the covariate, while changes relative to baseline were made with the Wilcoxon matched-pairs test. Histological scores were normalized by rank transformation before analysis. Regression analysis was used to relate the fall in extradural temperature to histological scores and the secondary rise in cortical impedance. All data are presented as mean \pm SEM.

Pilot studies. A pair of fetal sheep received scalp cooling for $48 \mathrm{~h}$ only. Whereas the first, minimally cooled (extradural temperature $35.9^{\circ} \mathrm{C}$ ) fetus developed very intense cortical seizure activity, the second fetus was better cooled (extradural temperature $32.0^{\circ} \mathrm{C}$ ) and developed brief epileptiform activity. However, when cooling was stopped in the second fetus there was reoccurrence of epileptiform activity, without a rise in cortical impedance. In view of this rebound hyperactivity, we decided that the fetuses in the hypothermia group should be cooled for $72 \mathrm{~h}$, for the main study.

To measure the effect of head cooling on the inner structures of 
the brain, another fetus was instrumented as above, but with two additional cerebral thermistors. Each thermistor was advanced through a burr hole $0.5 \mathrm{~cm}$ lateral to the midline, through the dura mater. The first was advanced $2.5 \mathrm{~cm}$ (corresponding with the depth of the hippocampus) and the second, $1 \mathrm{~cm}$. After $3 \mathrm{~d}$ of recovery, this fetus was cooled for $6 \mathrm{~h}$. The extradural temperature reached a stable temperature of $36.0^{\circ} \mathrm{C}$; the other three thermistors also indicated a stable temperature gradient; at $1 \mathrm{~cm}$ the temperature was $36.9^{\circ} \mathrm{C}$, and at $2.5 \mathrm{~cm}$ the temperature was $37.7^{\circ} \mathrm{C}$, while the core temperature was $38.4^{\circ} \mathrm{C}$.

\section{Results}

16 fetal sheep completed the full protocol: 9 randomized to the hypothermia group and 7 to the sham cooling group. In addition, three pilot studies were performed, as described in Methods. Differences between the hypothermia and sham groups for mean gestational age at experiment (124.7 \pm 1.3 vs. $123.2 \pm 1.4 \mathrm{~d})$, postmortem fetal weight $(3,920 \pm 250$ vs. $3,821 \pm$ 175 grams), baseline blood gases, $\mathrm{pH}$, glucose, lactate, blood pressure, fetal heart rate, and $\mathrm{CBF}$ were not significant.

Cooling was associated with a significant fall in extradural temperature, to a minimum of $32.4 \pm 0.8^{\circ} \mathrm{C}$ (vs. $39.1 \pm 0.04, P<$ 0.001 ) after $121 \pm 29 \mathrm{~min}$ of cooling; the time sequence of changes is shown in the top panel of Fig. 1. There was a smaller fall in esophageal temperature, reaching a trough of $37.1 \pm 0.5^{\circ} \mathrm{C}$ (vs. $38.8 \pm 0.2^{\circ} \mathrm{C}, P<0.01$ ) after $171 \pm 58 \mathrm{~min}$. The extradural and core temperatures in the hypothermia group

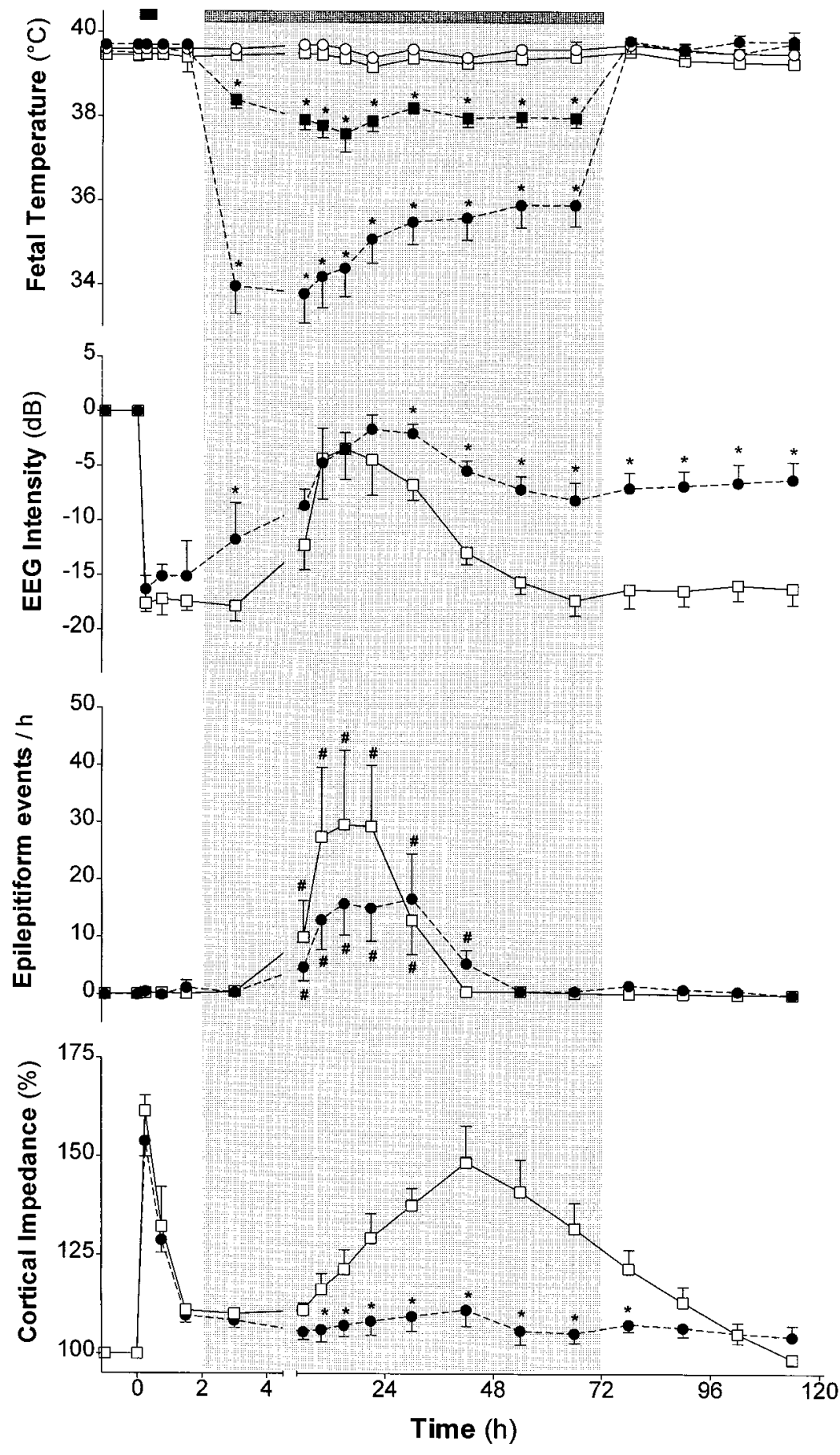

Figure 1. Time sequence of changes in fetal temperature and neurophysiological variables. The 30-min period of cerebral ischemia is shown by the solid bar, while cooling is shown by the gray bar, and the highlighted area. The top panel shows changes in extradural (filled circles) and esophageal (filled boxes) temperature in the hypothermia group and extradural (open circles) and esophageal (open squares) temperature in the sham cooled group. The lower three panels show changes in EEG intensity, epileptiform events detected (per hour), and cortical impedance (expressed as percentage of baseline) in the hypothermia (filled circles) and sham cooled (open boxes) groups. The hypothermia group shows greater final recovery of EEG intensity and complete suppression of the secondary rise in impedance, despite no significant effect on seizure activity. Mean \pm SEM, $* P<0.01$ hypothermia vs. sham cooled fetuses. ${ }^{\#} P<0.05$ vs. baseline. 


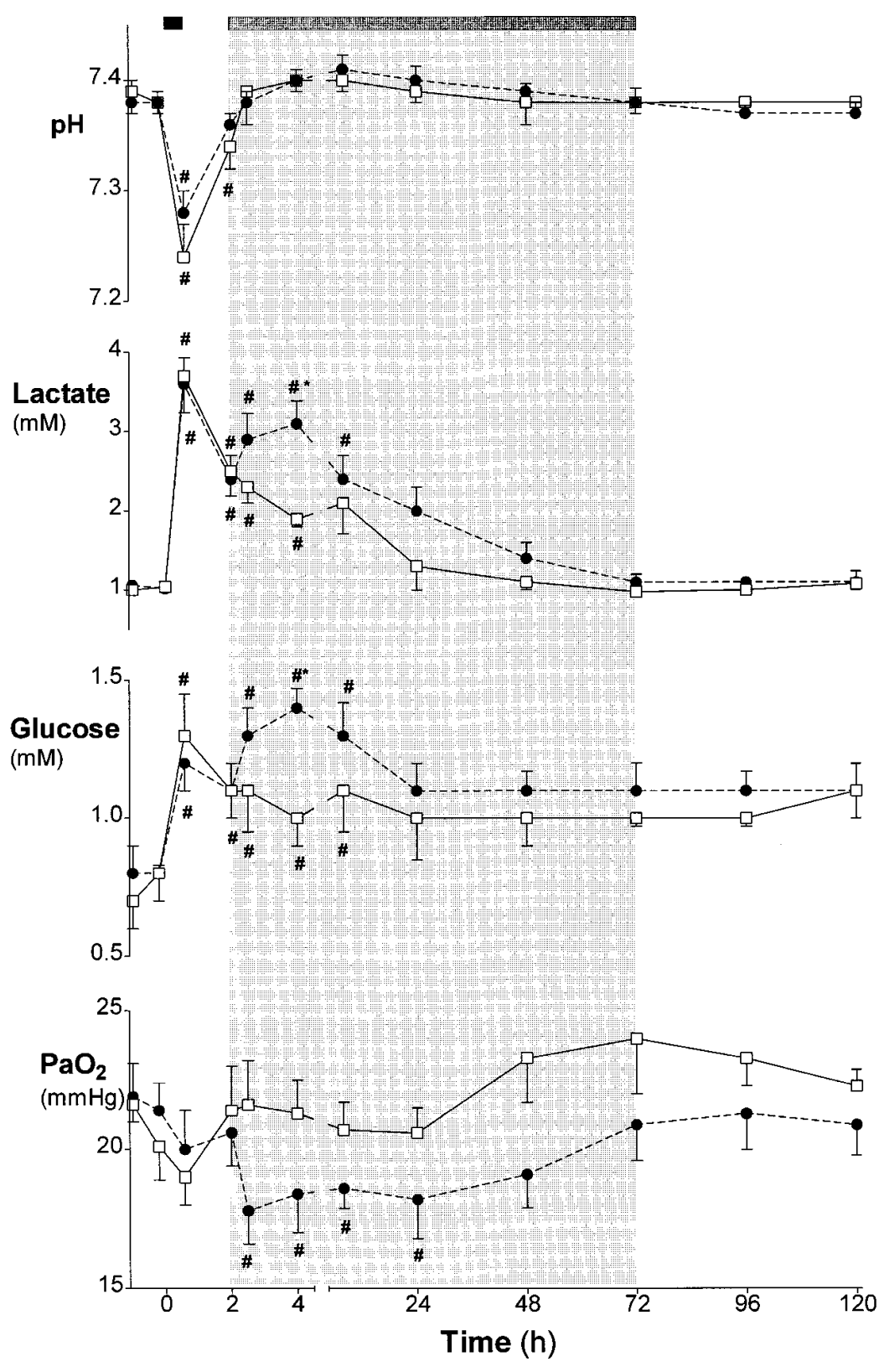

Figure 2. Time sequence of changes in fetal $\mathrm{pH}$, lactate, glucose, and $\mathrm{PaO}_{2}$ in the hypothermia (filled circles) and sham cooled groups (open squares). The period of cerebral ischemia is shown by the solid bar, while cooling is shown by the gray bar and the highlighted region. Both groups show a metabolic acidosis 5 min after reperfusion; the cooled group shows a transient rise in glucose and lactate, and a fall in $\mathrm{PaO}_{2}$ after the start of cooling. Mean \pm SEM, ${ }^{*} P<0.05$, hypothermia vs. controls, ${ }^{\#} P<0.05$ vs. baseline. rose during the second and third days of cooling but remained significantly lower than in the sham cooled fetuses throughout the whole period of cooling; the extradural temperature was $<34^{\circ} \mathrm{C}$ for $15.1 \pm 7 \mathrm{~h}$. In contrast, no significant change in maternal temperature was seen in either group $\left(38.3 \pm 0.3^{\circ} \mathrm{C}\right.$ vs. $\left.38.4 \pm 0.2^{\circ} \mathrm{C}, \mathrm{NS}\right)$.

The time sequence of changes in fetal arterial $\mathrm{pH}, \mathrm{PaO}_{2}$, glucose, and lactate levels is shown in Fig. 2. Cooling was associated with a significant interaction between treatment and time $(P<0.005)$ for fetal $\mathrm{PaO}_{2}$, lactate, and glucose, but not for $\mathrm{pH}$ or $\mathrm{PCO}_{2}$. Post-hoc testing suggested that the hypothermia, but not the sham cooled group, showed a small fall in $\mathrm{PaO}_{2}$ (greatest 30 min after the start of cooling) and a small secondary rise in lactate and glucose levels at $4 \mathrm{~h}$ (i.e., $2 \mathrm{~h}$ after the start of cooling).

Bilateral carotid artery occlusion for $30 \mathrm{~min}$ resulted in a pattern of suppression of EEG activity for many hours after reperfusion, followed by a rapid transition to high intensity, low frequency epileptiform activity, followed by resolution to the final level of EEG intensity (Fig. 1, second panel). The time sequence of changes in EEG intensity was significantly different in the two groups (ANOVA, $P<0.001$ ). The initial pattern of suppression followed by seizures was similar in the two groups, although the hypothermia group showed greater EEG intensity between 2 and $4 \mathrm{~h}(P<0.02)$. Time series analysis of the recorded signal showed that the time for EEG intensity to recover after reperfusion was $10.8 \pm 2.9 \mathrm{~h}$ in the hypothermia group compared with $10.8 \pm 3.0 \mathrm{~h}$ in sham cooled fetuses, while the EEG intensity peaked at $5.6 \pm 1.8$ vs. $5.0 \pm 2.2$ $\mathrm{dB}$ above baseline, after $19.7 \pm 2.6$ vs. $13.7 \pm 2.9 \mathrm{~h}$ (hypothermia versus sham cooled, NS). In contrast, the hypothermia group showed significantly greater EEG intensity from 36 to $120 \mathrm{~h}$; the 
final EEG intensity was $-5.2 \pm 1.6$ compared with $-15.5 \pm 1.5 \mathrm{~dB}$ in the sham cooled fetuses $(P<0.001)$.

Consistent with the changes in EEG intensity, off-line seizure detection analysis suggested an abrupt onset of epileptiform events, starting between 4 and $8 \mathrm{~h}$, and resolving between 24 and $36 \mathrm{~h}$ in both groups (Fig. 1, third panel). The rate of epileptiform events in the two groups was not significantly different.

The time sequence of changes in the $90 \%$ spectral edge showed a significant interaction between time and treatment group $(P<0.05)$. Baseline levels were similar in the hypothermia and sham groups $(13.3 \pm 0.4$ vs. $12.9 \pm 0.4 \mathrm{~Hz}, \mathrm{NS})$. The spectral edge fell after occlusion to similar levels in the two groups. The spectral edge was higher in the hypothermia group compared with the sham cooled group between 8 and $24 \mathrm{~h}$, corresponding with the phase of EEG hyperexcitability (8.9 \pm 0.6 vs. $5.2 \pm 0.6 \mathrm{~Hz}, P<0.05)$, then gradually rose in both groups. However, the spectral edge was significantly higher in the hypothermia group only in the last $24 \mathrm{~h}$ of recovery $(10.0 \pm 0.7$ vs. $7.0 \pm 0.5, P<0.05)$.

The time sequence of changes in cortical impedance is shown in the bottom panel of Fig. 1. Cortical impedance rose during occlusion, peaking at $157 \pm 5$ vs. $161 \pm 4 \%$ of baseline (hypothermia group vs. sham cooled group, NS) a few minutes after reperfusion, then declining to a nadir of $110 \pm 2$ vs. $111 \pm 1 \%$ (NS), $27 \pm 1$ vs. $30 \pm 3$ min (NS) after reperfusion. In the sham cooled fetuses, cortical impedance subsequently rose, with the onset of epileptiform activity, to a second peak of $152 \pm 8 \%, 39.1 \pm 1.4 \mathrm{~h}$ after reperfusion. In contrast, the rise was not seen in the hypothermia group (ANOVA, $P<0.001$ ); the highest measurement between 4 and $72 \mathrm{~h}$ was just $115 \pm 4 \%$ of baseline, not significantly higher than the residual impedance levels. When individual hypothermia animals were examined, a clear secondary peak was seen in only three out of nine cooled fetuses (compared with 7/7 sham cooled fetuses, $P=$ 0.01 ). These three fetuses were the least cooled within the hypothermia group: the extradural temperature was never suppressed to $<34^{\circ} \mathrm{C}$ in two, and only briefly in one.

There was no overall difference in the time sequence of changes in fetal heart rate and blood pressure between the groups (ANOVA). Both groups showed a significant hypertension during ischemia $(65.5 \pm 2.7$ vs. $65.0 \pm 3.5 \mathrm{mmHg}$, NS) which resolved over the hour after reperfusion. There was an apparent rise in blood pressure in the first $24 \mathrm{~h}$ of cooling, compared with baseline, which was not seen in the sham cooled fetuses (hypothermia group $53.3 \pm 1.7$ vs. $48.2 \pm 1.3$ $\mathrm{mmHg}, P<0.05$; sham cooled group $49.6 \pm 2.2$ vs. $48.2 \pm 1.3$ $\mathrm{mmHg}$, NS). This elevation subsequently resolved to baseline values despite continued cooling, and fetal blood pressure recordings remained normal in both groups until the end of the experiment.

The time sequence of changes in CBF showed a significant interaction between treatment and time (Fig. 3, top, $P<$ 0.001). CBF returned to normal immediately after reperfusion in all but one (sham cooled) fetus. After normalization, CBF fell abruptly between 48 and 174 min later (hypothermia group: $98 \pm 3$ min versus sham cooled group: $88 \pm 8 \mathrm{~min}, \mathrm{NS})$ to a minimum of $62 \pm 4$ vs. $65 \pm 6 \%$ of baseline (hypothermia versus sham cooled, NS). This secondary hypoperfusion persisted for $1-9 \mathrm{~h}(6.2 \pm 1.0$ vs. $3.9 \pm 0.9 \mathrm{~h}, P=0.06)$. CBF then progressively increased, peaking after $34.3 \pm 2.7$ vs. $28.7 \pm 2.8 \mathrm{~h}(\mathrm{NS})$ at $118 \pm 6$ vs. $141 \pm 6 \%$ of baseline (hypothermia versus sham cooled, $P=0.02$ ). Subsequently, the CBF of the hypothermia group remained higher than baseline levels, whereas in the sham cooled fetuses, CBF fell below baseline values.

$\mathrm{CMRO}_{2}$ was able to be calculated in five hypothermia and five sham cooled fetuses (Fig. 3, bottom), up to $8 \mathrm{~h}$ after occlu-

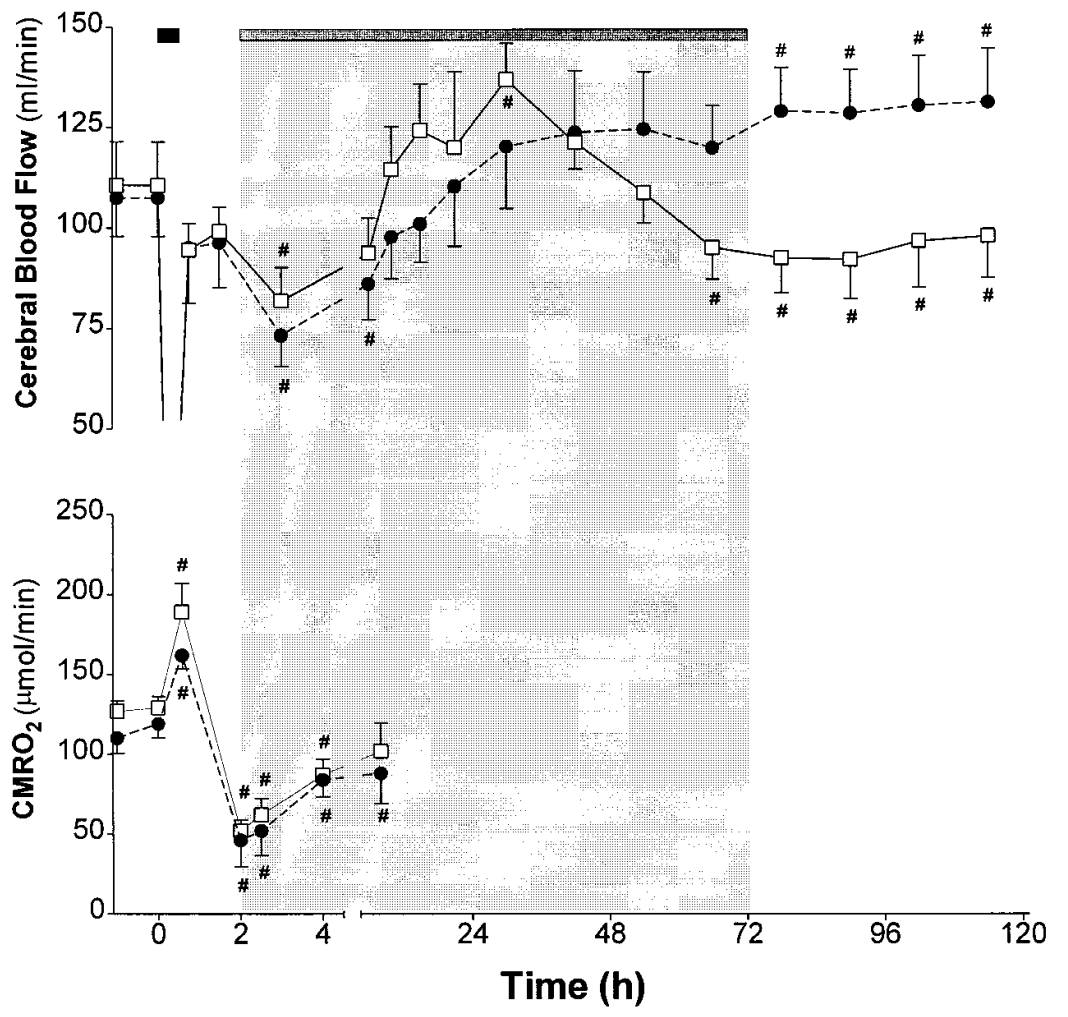

Figure 3. Time sequence of changes in total CBF $(\mathrm{ml} / \mathrm{min})$ and $\mathrm{CMRO}_{2}(\mu \mathrm{mol} / \mathrm{min})$ in the hypothermia (filled circles) and sham cooled (open boxes) groups. Cerebral ischemia is shown by the solid bar at the top of the graph, while cooling is shown by the gray bar, and by the highlighted region. CBF but not $\mathrm{CMRO}_{2}$ showed a significant interaction between cooling and time $(P<0.001$, ANOVA). Mean \pm SEM, ${ }^{\#} P<0.05$ vs. baseline. 5 min after reperfusion there was an increase in $\mathrm{CMRO}_{2}$, due to increased cerebral oxygen extraction. In contrast, the phase of secondary hypoperfusion, between 2 and $8 \mathrm{~h}$, was paralleled by suppression of $\mathrm{CMRO}_{2}$ in both groups. 

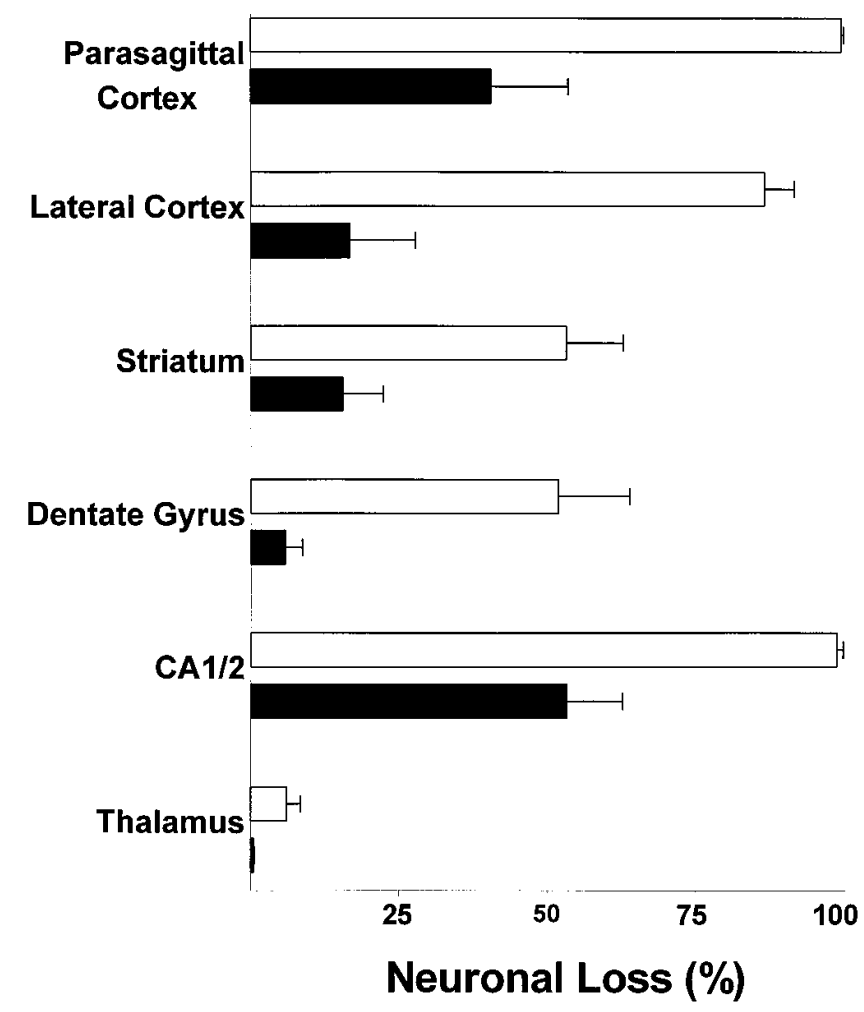

Figure 4. Effect of $72 \mathrm{~h}$ of cerebral cooling started $90 \mathrm{~min}$ after reperfusion on microscopically assessed neuronal loss in different brain regions at $5 \mathrm{~d}$ after ischemia. A significant reduction $(P<0.001)$ in neuronal loss was seen in all regions in fetuses treated with selective cerebral cooling (closed bars) compared with sham cooled fetuses (open bars). Mean \pm SEM.

sion. There was no significant difference between the groups over this time period. In both groups there was a rise in metabolic rate $5 \mathrm{~min}$ after reperfusion. This was associated with similar CBF to baseline and an increase in the difference between the arterial and venous oxygen contents, compared with baseline $(P<0.05) . \mathrm{CMRO}_{2}$ then fell to a nadir at $2 \mathrm{~h}(90 \mathrm{~min}$ after reperfusion) and then progressively rose up to $8 \mathrm{~h}$. This fall was due to the secondary fall in $\mathrm{CBF}$ discussed above; cerebral oxygen extraction was not significantly different from baseline at these times.

Neuronal loss scores, across all the regions assessed, were significantly lower in the hypothermia group compared with the sham cooled group $(P<0.001$, Fig. 4$)$. The extent of infarction in the parasagittal cortex was markedly reduced in the cooled group compared with the sham cooled group (29 \pm 13 vs. $100 \pm 0 \%, P<0.001)$. Examples of the microscopic changes are shown in Figs. 5 and 6. When residual impedance was included as a covariate as a measure of the "severity" of the insult, it was also independently correlated with outcome $(r=$ $0.6, P<0.02$, for parasagittal cortex neuronal loss scores). However, this adjustment did not alter the effect of hypothermia $(P<0.001)$. Within the hypothermia group alone, the extradural temperature, measured between 4 and $8 \mathrm{~h}$, was correlated with both the secondary peak impedance $\left(r^{2}=0.62, P<\right.$ $0.001)$ and the histological score in the parasagittal cortex $\left(r^{2}=\right.$ $0.42, P<0.03)$.

\section{Discussion}

Moderate, selective cerebral hypothermia, starting $90 \mathrm{~min}$ after reperfusion and continuing for $3 \mathrm{~d}$, dramatically reduced neuronal loss in all areas assessed and nearly abolished cortical infarction. This was associated with greater residual EEG activity after $5 \mathrm{~d}$ of recovery and suppression of secondary cortical edema. Despite these beneficial effects, it did not prevent delayed epileptiform activity, nor did it significantly alter cerebral oxidative metabolism before the onset of seizures. The selective head cooling, causing a fall in core temperature of $1-3^{\circ} \mathrm{C}$, was associated with only modest systemic effects. There was a small and transient fall in $\mathrm{PaO}_{2}$ and rise in glucose, lactate, and fetal blood pressure, consistent with our previous studies of systemic hypothermia in utero (19). The use of a chronically instrumented fetal preparation avoids the cerebral metabolic effects of anesthesia (25) which might interact with hypothermia and ensures stable control temperatures (19).

The present data suggest that substantial neuroprotection was seen only in fetuses in whom there was a sustained fall of the extradural temperature to $\sim 34^{\circ} \mathrm{C}$ or less. This suggests that there is a critical depth of hypothermia required for effective neuronal rescue. This is likely to be modified by the severity of the initial insult, by how soon hypothermia is started after the insult, and by how long cooling is continued $(6,7,11,12)$.

Immediate cooling after hypoxia-ischemia will seldom be practical in the clinical setting. Despite a delay in initiating cooling of $90 \mathrm{~min}$, plus the time taken for the brain to cool, hypothermia was still effective in reducing damage in the present study, confirming that considerable damage does occur many hours after the insult. 5 min after reperfusion, $\mathrm{CMRO}_{2}$ was higher than baseline, with normal $\mathrm{CBF}$, but increased oxygen extraction, consistent with relative limitation of oxygen delivery in a situation of oxygen debt. These data, taken with the observation that the cortical impedance also did not begin to fall for 5-10 min after reperfusion, suggest that there is still ongoing primary damage at this time (15). This may be one reason why studies of brief hypothermia have tended to show neuroprotection only if started immediately after reperfusion $(8,9)$. In effect, this strategy would represent intervention late in the primary phase.

The critical duration of cerebral cooling required for significant neuronal rescue is unknown. In this study, rebound epileptiform activity was seen in a pilot experiment when cooling was stopped after $48 \mathrm{~h}$, whereas it was not seen in any fetus after $72 \mathrm{~h}$ cooling. The few studies which have systematically addressed this also suggest that prolonged cooling is needed (7, $10,12)$ and that this critical period is longer, the more severe the original insult (7). In a previous report where unanesthetized infant rats were subjected to a moderate hypoxic-ischemic insult followed by mild hypothermia $\left(2-3^{\circ} \mathrm{C}\right.$ cerebral cooling), hypothermia needed to be continued for $3 \mathrm{~d}$ to reduce cortical infarction; shorter periods were ineffective (11).

There have been reports, from studies of brief cooling, that hypothermia only delayed rather than prevented neuronal degeneration (26). Such studies have generally addressed models of mild ischemia producing very delayed selective neuronal loss in the hippocampus, rather than severe loss with infarction. In contrast, the effectiveness of prolonged postinsult hypothermia in the infant rat, in preventing cerebral infarction after hypoxia-ischemia, was unchanged after 3 wk of recovery (11). In this study, we allowed the fetal sheep to recover for a 

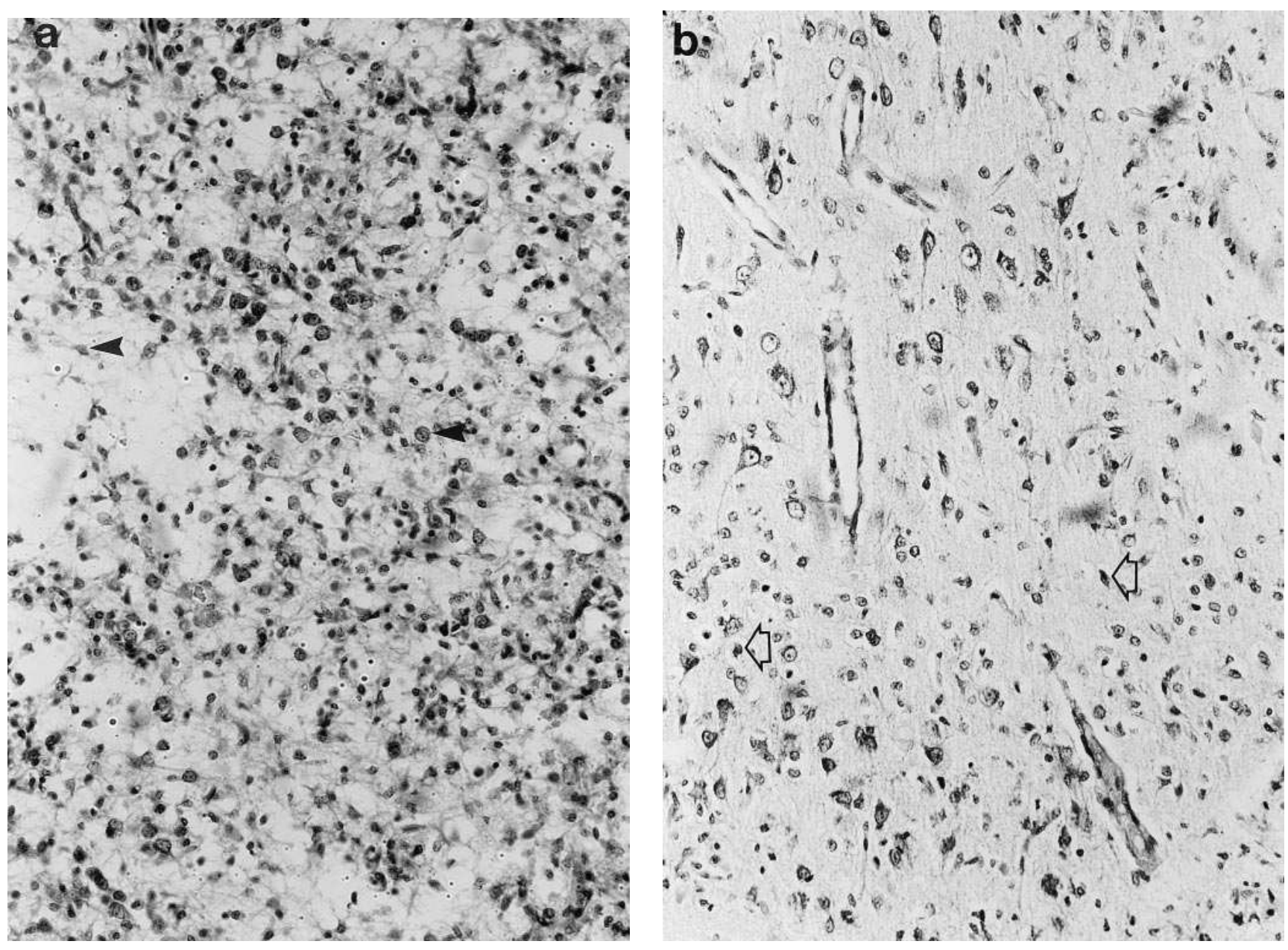

Figure 5. Photomicrographs $(\times 370)$ of the parasagittal parietal cortex in sham cooled $(a)$ and hypothermia $(b)$ fetuses. The brains were perfusion fixed $5 \mathrm{~d}$ after ischemia. The sham cooled cortex shows infarction, with disruption of the cytoarchitecture. No viable neurons are seen, and there is a marked microglial reaction (solid arrows). The cortex of the cooled fetus $(b)$ shows preservation of the cytoarchitecture. There are only a few dead neurons, which are surrounded by normal, surviving cells. Neurons showing ischemic cell change with pyknosis of the nuclei and cytoplasmic eosinophilia are shown (open arrows). Thionin and acid fuchsin stain.

further $48 \mathrm{~h}$ after the end of cooling. During this time EEG activity continued to gradually improve, and there was no suggestion of a further "tertiary" deterioration in any biophysical parameter measured.

The mechanism of hypothermic neuroprotection is not established. In principle, hypothermia might work by $(a)$ reducing cellular metabolic demands, and so delaying neuronal depolarization; $(b)$ reducing the excessive accumulation of excitotoxins such as glutamate, and so reducing intracellular calcium accumulation; or $(c)$ suppressing the toxic consequences of these events, such as activation of endonucleases or production of oxygen free radicals.

Hypothermia produces a graded reduction in cerebral metabolism (27); the resulting reduction in the rate of depletion of high energy metabolites during hypoxia-ischemia is likely to make a substantial contribution to its neuroprotective effects. In contrast, in this study hypothermia had no significant effect on the already suppressed $\mathrm{CBF}$ and $\mathrm{CMRO}_{2}$ after ischemia, before the onset of seizures. It is possible that there may have been an effect on $\mathrm{CMRO}_{2}$ during the subsequent seizure activity, since there was a significant reduction in peak secondary hyperperfusion with cooling. However, there was only a trend to a reduction in numbers of seizures, while the peak of seizure intensity was similar. Consistent with these data, hypothermia is reported to delay neuronal depolarization significantly more than general anesthesia for a given reduction of cerebral metabolism, suggesting that suppression of metabolism alone cannot explain even its immediate cerebral effects (25). The increase in CBF during the resolution phase in cooled fetuses, compared with the decrease associated with sham cooling, was probably related to reduced damage rather than a direct cooling effect. In earlier studies using near infrared spectroscopy, there was an inverse correlation between vasodilation in this phase and neuronal loss (17).

Accumulation of excitatory amino acids (excitotoxins) during ischemia (28) or in association with seizures after ischemia (16) is suggested to be one factor causing immediate damage and triggering the cascade leading to secondary neuronal death. The well known rise in excitotoxins during ischemia is suppressed by mild to moderate hypothermia, both in vitro $(28)$ and in vivo $(2,29)$. In a microdialysis study using the same experimental approach as in this report, we found a large rise 

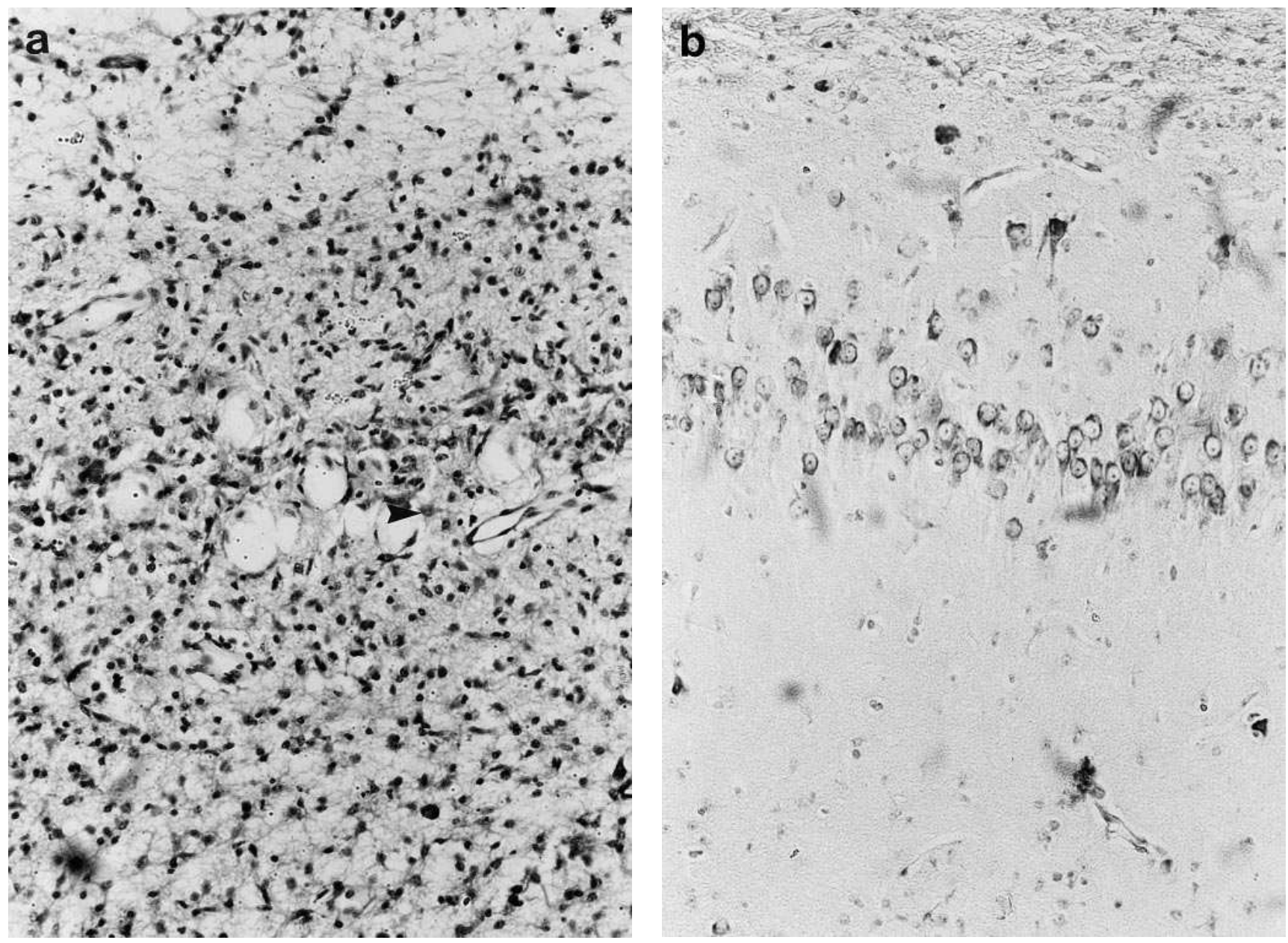

Figure 6. Photomicrographs $(\times 370)$ of the CA1 region of the hippocampus from a control fetus $(a)$ and a cooled fetus $(b)$. The control hippocampus shows marked stromal edema with no surviving neurons seen. There is a marked glial reaction (solid arrow). The hippocampus of the cooled fetus shows preservation of the cytoarchitecture, with no ischemic cell death. Thionin and acid fuchsin stain.

in the ratio of excitatory to inhibitory neurotransmitters in the parietal cortex during the secondary phase (16). This rise paralleled the secondary rise in cortical impedance, rather than seizure activity, suggesting that the excessive accumulation of excitotoxins was related to cell membrane dysfunction. The dramatic suppression of impedance in this study might then be consistent with reduced accumulation of excitotoxins.

Suppression of excitotoxin accumulation cannot fully account for hypothermic neuroprotection, since moderate hypothermia had no effect on the rise in excitotoxins after head injury in the rat (30), and yet markedly reduced subsequent damage. Consistent with this, in vitro hypothermia had no immediate effect on glutamate receptor activity or its toxic effects (28). In contrast, prolonged hypothermia, applied after the excitotoxins had been washed out, was protective $(28,31)$. Furthermore, hypothermia during cardiac arrest in the rat prolonged anoxic depolarization despite having no effect on the transfer of calcium from extracellular fluid into cells (32). These data suggest that the neuroprotective effect of hypothermia may be primarily mediated by reducing the effects of ischemia on second or third messengers and on production of other cytotoxic agents (3).

Beyond their association with excitotoxins, postasphyxial seizures may themselves be damaging, as status epilepticus can cause hypermetabolic neuronal loss (33). In this study hypothermia was effective in reducing cerebral damage and prevented secondary cytotoxic edema, without significantly reducing postinsult epileptiform activity. In contrast, the highly selective glutamate antagonist MK-801, given from 6 to $24 \mathrm{~h}$ after reperfusion, totally abolished seizure activity (34); despite this, a secondary rise in impedance occurred, although delayed compared with sham treated fetuses, and parasagittal damage was not reduced. These data clearly demonstrate that the processes contributing to secondary membrane dysfunction and to seizures are distinct, and suggest that postasphyxial seizures make a relatively small direct contribution to cortical infarction.

It has been reported that hypothermia is able to prevent apoptotic or programmed cell death, but not necrotic cell death after hypoxic-ischemic injury (35). This is an attractive concept, since by analogy with the normal process of apoptosis during neurodevelopment, it would suggest that hypothermia acts by suppressing a predefined cascade of intracellular events (36). However, a detailed study of the time course of neuronal death after hypoxia-ischemia in the developing brain found that selective neuronal death was predominantly apoptotic, 
but that cell death during the evolution of infarction was associated with a necrotic morphology (37). Thus this study, in which delayed hypothermia prevented the development of cortical infarction or pannecrosis, does not support such a specific mode of action.

In conclusion, delayed moderate cerebral hypothermia, maintained throughout the secondary phase of cerebral dysfunction, dramatically improved neural outcome from a severe ischemic insult in fetal sheep. The mild core cooling was associated with only small, transient systemic effects, and hypotension did not occur. Hypothermia prevented secondary cell membrane dysfunction, despite having no significant effect on either cerebral metabolism or seizure activity. These data are consistent with the hypothesis that hypothermia acts on intracellular cytotoxic mechanisms in the secondary phase of damage, rather than by reducing oxidative metabolism or neuronal overexcitation. Selective head cooling may prove to be a safe and effective therapy for moderate to severe hypoxic-ischemic encephalopathy in term infants.

\section{Acknowledgments}

This research was supported by U.S. Public Health Service grant R01-HD-32752, the Auckland Medical Research Foundation, the New Zealand Lottery Grants Board, the Niels Stensen Stichting (Amsterdam, The Netherlands), and the Health Research Council of New Zealand.

\section{References}

1. Busto, R., W.D. Dietrich, M.Y. Globus, I. Valdes, P. Scheinberg, and M.S. Ginsberg. 1987. Small differences in intra ischemic brain temperature critically determine the extent of ischemic neuronal injury. J. Cereb. Blood Flow Metab. 7:729-738.

2. Illievich, U.M., M.H. Zornow, K.T. Choi, M.S. Scheller, and M.A.P. Strnat. 1994. Effects of hypothermic metabolic suppression on hippocampal glutamate concentrations after transient global cerebral ischemia. Anesth. Analg. 78:905-911.

3. Karibe, H., S.F. Chen, G.J. Zarow, J. Gafni, S.H. Graham, P.H. Chan, and P.R. Weinstein. 1994. Mild intraischemic hypothermia suppresses consumption of endogenous antioxidants after temporary focal ischemia in rats. Brain Res. 649:12-18.

4. Yager, J., J. Towfighi, and R.C. Vannucci. 1993. Influence of mild hypothermia on hypoxic-ischemic brain damage in the immature rat. Pediatr. Res. 34:525-529.

5. Thoresen, M., R. Bagenholm, E.M. Loberg, F. Apricena, and I. Kjellmer. 1996. Posthypoxic cooling of neonatal rats provides protection against brain injury. Arch. Dis. Child. 74:F1-F7.

6. Chopp, M., H. Chen, M.O. Dereski, and J.H. Garcia. 1991. Mild hypothermic intervention after graded ischemic stress in rats. Stroke. 22:37-43.

7. Colbourne, F., and D. Corbett. 1994. Delayed and prolonged post-ischemic hypothermia is neuroprotective in the gerbil. Brain Res. 654:265-272.

8. Busto, R., W.D. Dietrich, M.Y. Globus, and M.D. Ginsberg. 1989. Postischemic moderate hypothermia inhibits CA1 hippocampal ischemic neuronal injury. Neurosci. Lett. 101:299-304.

9. Kuboyama, K., P. Safar, A. Radovsky, S.A. Tisherman, S.W. Stezoski, and H. Alexander. 1993. Delay in cooling negates the beneficial effect of mild resuscitative cerebral hypothermia after cardiac arrest in dogs: a prospective, randomized study. Crit. Care Med. 21:1348-1358.

10. Carroll, M., and O. Beek. 1992. Protection against hippocampal CA1 cell loss by post-ischemic hypothermia is dependent on delay of initiation and duration. Metab. Brain Dis. 7:45-50.

11. Sirimanne, E.S., R.M. Blumberg, D. Bossano, M.I. Gunning, A.D. Edwards, P.D. Gluckman, and C.E. Williams. 1996. The effect of prolonged modification of cerebral temperature on outcome following hypoxic ischemic injury in the infant rat. Pediatr. Res. 39:591-598.

12. Coimbra, C., and T. Wieloch. 1994. Moderate hypothermia mitigates neuronal damage in the rat brain when initiated several hours following transient cerebral ischemia. Acta Neuropathol. 87:325-331.

13. Thoresen, M., J. Penrice, A. Lorek, E.B. Cady, M. Wylezinska, V. Kirkbride, C.E. Cooper, G.C. Brown, A.D. Edwards, J.S. Wyatt, and E.O. Reynolds. 1995. Mild hypothermia after severe transient hypoxia-ischemia ameliorates delayed cerebral energy failure in the newborn piglet. Pediatr. Res. 37:
667-670.

14. Roth, S.C., A.D. Edwards, E.B. Cady, D.T. Delpy, J.S. Wyatt, D. Azzopardi, J. Baudin, J. Townsend, A.L. Stewart, and E.O. Reynolds. 1992. Relation between cerebral oxidative metabolism following birth asphyxia, and neurodevelopmental outcome and brain growth at one year. Dev. Med. Child Neurol. 34:285-295.

15. Williams, C.E., A.J. Gunn, and P.D. Gluckman. 1991. The time course of intracellular edema and epileptiform activity following prenatal cerebral ischemia in sheep. Stroke. 22:516-521.

16. Tan, W.K.M., C.E. Williams, M.J. During, C.E. Mallard, M.I. Gunning, A.J. Gunn, and P.D. Gluckman. 1996. Accumulation of cytotoxins during the development of seizures and edema after hypoxic-ischemic injury in late gestation fetal sheep. Pediatr. Res. 39:791-797.

17. Marks, K.A., E.C. Mallard, I. Roberts, C.E. Williams, E.S. Sirimanne, B.M. Johnston, P.D. Gluckman, and A.D. Edwards. 1996. Delayed vasodilation and altered oxygenation following cerebral ischemia in fetal sheep. Pediatr. Res. 39:48-54.

18. Gunn, T.R., and E.W. Outerbridge. 1978. Effectiveness of neonatal transport. Can. Med. Assoc. J. 118:646-649.

19. Gunn, T.R., J.H. Butler, and P.D. Gluckman. 1986. Metabolic and hormonal responses to cooling the fetal lamb in utero. J. Dev. Physiol. 8:55-66.

20. Gunn, A.J., C.E. Williams, E.C. Mallard, W.K.M. Tan, and P.D. Gluckman. 1994. Flunarizine, a calcium channel antagonist, is partially prophylactically neuroprotective in hypoxic-ischemic encephalopathy in the fetal sheep. Pediatr. Res. 35:657-663.

21. Van Bel, F., C. Roman, R.J.M. Klautz, D.F. Teitel, and A.M. Rudolph. 1994. Relationship between brain blood flow and carotid arterial flow in the sheep fetus. Pediatr. Res. 35:329-333.

22. Johnston, B.M., E.C. Mallard, C.E. Williams, and P.D. Gluckman. 1996. Insulin-like growth factor-1 is a potent neuronal rescue agent following hypoxic-ischemic injury in fetal lambs. J. Clin. Invest. 97:300-308.

23. Gasser, T., P. Bacher, and J. Mocks. 1982. Transformations towards the normal distribution of broad band spectral parameters of the EEG. Electroencephalogr. Clin. Neurophysiol. 53:119-124.

24. Smith, D.C. 1992. Effects of skin blood flow and temperature on skin: electrode impedance and offset potential: measurements at low alternating current density. J. Med. Eng. Technol. 16:112-116.

25. Nakashima, K., M.M. Todd, and D.S. Warner. 1995. The relation between cerebral metabolic rate and ischemic depolarization. A comparison of the effects of hypothermia, pentobarbital, and isoflurane. Anesthesiology. 82: $1199-1208$.

26. Dietrich, W.D., R. Busto, O. Alonso, M.Y. Globus, and M.D. Ginsberg. 1993. Intraischemic but not postischemic brain hypothermia protects chronically following global forebrain ischemia in rats. J. Cereb. Blood Flow Metab. 13:541-549.

27. Laptook, A.R., R.J.T. Corbett, R. Sterett, D. Garcia, and G. Tollefsbol. 1996. Quantitative relationship between brain temperature and energy utilization rate measured in vivo using ${ }^{31} \mathrm{P}$ and ${ }^{1} \mathrm{H}$ magnetic resonance spectroscopy. Pediatr. Res. 38:919-925.

28. Bruno, V.M.G., M.P. Goldberg, L.L. Dugan, R.G. Giffard, and D.W. Choi. 1994. Neuroprotective effect of hypothermia in cortical cultures exposed to oxygen-glucose deprivation or excitatory amino acids. J. Neurochem. 63: 1398-1406.

29. Busto, R., M.Y. Globus, W.D. Dietrich, E. Martinez, I. Valdes, and M.D. Ginsberg. 1989. Effect of mild hypothermia on ischemia-induced release of neurotransmitters and free fatty acids in rat brain. Stroke. 20:904-910.

30. Palmer, A.M., D.W. Marion, M.L. Botscheller, and E.E. Redd. 1993. Therapeutic hypothermia is cytoprotective without attenuating the traumatic brain injury-induced elevations in interstitial concentrations of aspartate and glutamate. J. Neurotrauma. 10:363-372.

31. Shuaib, A., E. Sochocka, W. Code, and L. Hertz. 1992. Hypothermia protects astrocytes during ischemia in cell culture. Neurosci. Lett. 146:69-71.

32. Kristian, T., K. Katsura, and B.K. Siesjö. 1992. The influence of moderate hypothermia on cellular calcium uptake in complete ischaemia: implications for the excitotoxic hypothesis. Acta Physiol. Scand. 146:531-532.

33. Nevander, G., M. Ingvar, R.N. Auer, and B.K. Siesjö. 1985. Status epilepticus in well-oxygenated rats causes neuronal necrosis. Ann. Neurol. 18:281290.

34. Tan, W.K.M., C.E. Williams, A.J. Gunn, E.C. Mallard, and P.D. Gluckman. 1992. Suppression of postischemic epileptiform activity with MK-801 improves neural outcome in fetal sheep. Ann. Neurol. 32:677-682.

35. Edwards, A.D., X. Yue, M.V. Squier, M. Thoresen, E.B. Cady, J. Penrice, C.E. Cooper, J.S. Wyatt, E.O. Reynolds, and H. Mehmet. 1995. Specific inhibition of apoptosis after cerebral hypoxia-ischaemia by moderate post-insult hypothermia. Biochem. Biophys. Res. Commun. 217:1193-1199.

36. Johnson, E.M., L.J.S. Greenlund, P.T. Akins, and C.Y. Hsu. 1995. Neuronal apoptosis: current understanding of molecular mechanisms and potential role in ischemic brain injury. J. Neurotrauma. 12:843-852.

37. Beilharz, E.J., C.E. Williams, M. Dragunow, E.S. Sirimanne, and P.D. Gluckman. 1995. Mechanisms of delayed cell death following hypoxic-ischemic injury in the immature rat: evidence for apoptosis during selective neuronal loss. Mol. Brain. Res. 29:1-14. 